\title{
Veículo Autônomo: Uma Visão Geral da Produção Científica baseada na Análise Bibliométrica
}

\author{
Vehículo Autónomo: Una Visión General de la Producción Científica basada \\ en el Análisis Bibliométrico
Autonomous Vehicle: An Overview of Scientific Production based on Bibliometric Analysis

Amanda Basilio Romano.

Ingeniera Civil, M.Sc(c) Transportes Urbanos.

Universidade de Brasília (UnB), Brasilia, Brasil.

amandaromano27@gmail.com

(iD) http://orcid.org/0000-0002-6524-8328

Pastor Willy Gonzales Taco.

Ingeniero Civil, M.Sc. Transportes Urbanos. Dr. en Ingeniería de Transportes.

Universidade de Brasília (UnB), Brasilia, Brasil.

pwgtaco@gmail.com

(iD) https://orcid.org/0000-0002-2055-9114

Recibido: diciembre 1 de 2020

Aceptado: enero 5 de 2021

Publicado: enero 10 de 2021

\section{RESUMO}

Este artigo tem como objetivo identificar o estado da arte dos estudos sobre Veículos Autônomos (AV), considerando o desenvolvimento do assunto e suas linhas de pesquisa, utilizando uma Revisão Bibliométrica nas bases de dados Web of Science e Scopus. Nos clusters, gerados pelo acoplamento bibliográfico, destacamse duas linhas de pesquisa, focadas nos aspectos tecnológicos e nos aspectos sociais e comportamentais da aceitabilidade da AV. Em relação aos países, Estados Unidos, Alemanha e China lideram o número de publicações referentes a VA nas duas bases.

Palavras-chave: Autonomous Vehicle; Análise Bibliométrica; VOSviewer.

\section{RESUMEN}

Este artículo tiene como objetivo identificar el estado del arte de los estudios sobre Vehículos Autónomos (VA), considerando el desarrollo del tema y sus líneas de investigación, utilizando una Revisión Bibliométrica en las bases de datos de Web of Science y Scopus. En los clusters, generados por el acoplamiento bibliográfico, se destacan dos líneas de investigación, centradas en los aspectos tecnológicos y en los aspectos sociales y comportamentales de la aceptabilidad de AV. En cuanto a los países, Estados Unidos, Alemania y China lideran el número de publicaciones referidas a AV en las dos bases de datos.

Palabras clave: Vehículo Autónomo; Análisis Bibliométrico; VOSviewer. 


\section{ABSTRACT}

This article aims to identify the state of the art of studies on Autonomous Vehicles (AV), considering the development of the subject and its lines of research, using a Bibliometric Review in the Web of Science and Scopus databases. In the clusters, generated by the bibliographic coupling, two lines of research stand out, focused on the technological aspects and on the social and behavioral aspects of the acceptability of VA. Regarding the countries, the United States, Germany and China lead the number of publications referring to VA in the two databases.

Keywords: Autonomous Vehicle; Bibliometric Analysis; VOSviewer.

\section{INTRODUÇÃO}

Na última década o desenvolvimento tecnológico tem apresentado uma serie de soluções para os vários problemas da mobilidade urbana e dos transportes, utilizando-se da Inteligência Artificial. O desenvolvimento tecnológico dos Veículos Autônomos (VAs), é uma dessas soluções que por apresentarem diversos benefícios, desvantagens, barreiras, e mudanças comportamentais, são necessários estudos para avaliar os requisitos da sua implantação nos centros urbanos.

Dentre os benefícios, tem-se a redução de acidentes, o aumento da mobilidade, a redução de congestionamentos, uma menor necessidade de estacionamentos, 0 incentivo a programas de compartilhamento, a melhoria na economia de combustível proporcionando a redução de emissões, o aumento da capacidade das vias, e uma operação mais eficiente dos veículos com melhor tempo de viagem (Anderson et al., 2014; Fagnant e Kockelman, 2015).

Como desvantagens estima-se que sua adoção gerará um aumento de milhas percorridas por veículos (VMT) (Anderson et al., 2014; Fagnant e Kockelman, 2015; Milakis et al,, 2017)the potential effects of automated driving that are relevant to policy and society are explored, findings discussed in literature about those effects are reviewed and areas for future research are identified. The structure of our review is based on the ripple effect concept, which represents the implications of automated vehicles at three different stages: first-order (traffic, travel cost, and travel choices, o que pode causar congestionamentos (Anderson et al., 2014), aumento de consumo de combustível (Anderson et al,, 2014; Fagnant e Kockelman, 2015) e consequentemente de emissões, com o aumento do número de viagens (Fagnant e Kockelman, 2015).

Procesos
Urbanos
As barreiras da implantação estão relacionadas com a tecnologia, tais como os ciberataques em potencial (Petit e Shladover, 2015), questões éticas sobre quem se responsabilizaria em caso de acidente envolvendo VA (Hevelke e Nida-Rümelin, 2015), e como devem ser configurados o ambiente de ética e programação dos VA para se comportarem no caso de um acidente inevitável (Gogoll e Müller, 2017; Santoni de Sio, 2017). Ainda a incorporação dos VAs causaria mudanças comportamentais que afetaria a maneira como os usuários interagiriam com os sistemas de transporte, pois os seus deslocamentos seriam feitos sem nenhuma intervenção humana na condução.

Como observado, a implantação de VAs no ambiente urbano, pode-se gerar diversos resultados, podendo contribuir na formação de cidades sustentáveis, recuperando espaço urbano, com menos veículos nas vias, como também reforçando uma rede de transporte público eficiente (The International Association of Public Transport, 2017).

Nesse contexto em que incorporação dos VAs vem precedido de um processo tecnológico dinâmico, é essencial conhecer o desenvolvimento do tema e identificar sua evolução e as potenciais tendências futuras. Assim, o artigo teve como objetivo identificar o estado da arte dos estudos sobre VAs, considerando o desenvolvimento do tema e suas linhas de pesquisa utilizando-se de uma Revisão Bibliométrica. Para isto, o artigo foi estruturado em quatro seções, após esta introdução tem-se a seção do método adotado de Revisão Bibliométrica. $\mathrm{Na}$ terceira seção foram apresentados os resultados obtidos da revisão bibliométrica sobre VA dos artigos indexados nas bases de dados da Web of Science e Scopus e na quarta seção, foram feitas as considerações finais. 


\section{Método da Revisão Bibliométrica}

A Revisão Bibliométrica (RB) é um método de análise quantitativa que possibilita a medição da contribuição do conhecimento científico das publicações, em artigos indexados em bases de dados ou eventos de áreas específicas de conhecimento. A RB pode contribuir para analisar a visão geral e perspectiva quantitativa de um tema específico, identificando os principais pontos e ideias emergentes de um campo de pesquisa, avaliando o desempenho e a influência de diferentes países ou periódicos, e observando a evolução e a tendência de desenvolvimento (Li et al., 2017).

A RB se foca em análise de redes tanto em documentos, palavras-chave, bem como em autores ou periódicos, usando técnicas de mapeamento e agrupamento, que são complementares entre si, com o objetivo de fornecer informações sobre a estrutura de uma rede, observando quais os tópicos ou campos de pesquisa, como se relacionam e como o domínio científico se desenvolveu ao longo do tempo (Waltman et al,, 2010).
Diversas aplicações podem ser vistas em estudos de RB na área de transporte e mobilidade urbana. Romano et al. (2018), utilizando a RB identificaram artigos relevantes para o uso da bicicleta, e assim levantaram os fatores que influenciam o uso da bicicleta em áreas urbanas; Modak et al. (2019) analisaram o estado da arte do conhecimento em transportes perante a RB das publicações da revista Transportation Research num período de 50 anos; Gandia et al. (2019) fizeram uma revisão cienciométrica e bibliométrica sobre o tema de VA. A estrutura do método de RB (Figura 1), inicia-se com a primeira etapa da preparação da pesquisa, definindo a string de pesquisa e suas delimitações. $\mathrm{Na}$ segunda etapa, a partir dos dados extraídos da pesquisa, foi possível observar a evolução das publicações e citações referente ao tema. $\mathrm{Na}$ terceira etapa foram apresentados os periódicos que mais publicaram e citaram sobre o tema. $\mathrm{Na}$ quarta etapa foram desenvolvidos mapas de acoplamento bibliográfico, para identificar as linhas de pesquisas sobre VAs, analisando-se os clusters. E na quinta etapa foi gerada a distribuição das publicações por países.

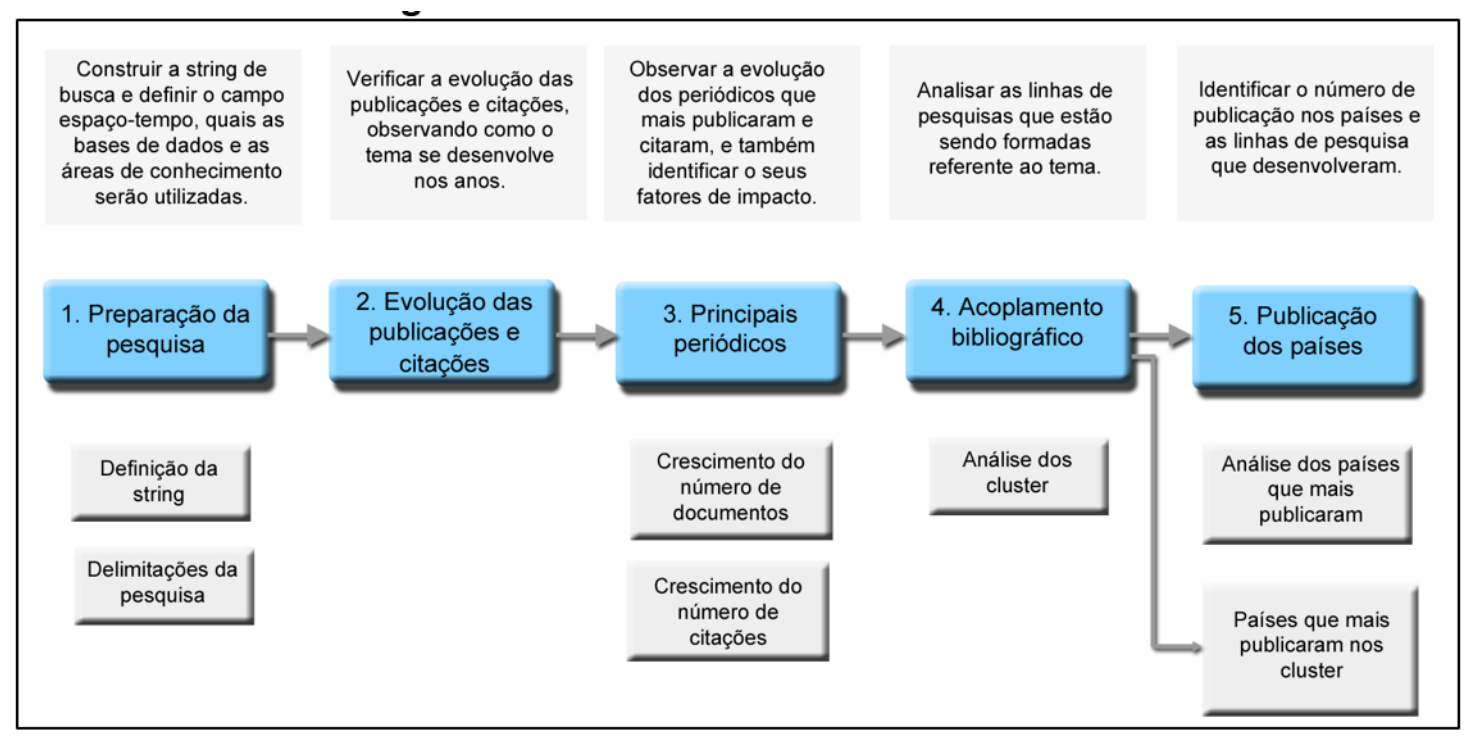

Figura 1. Método da Revisão Bibliométrica

Fonte: Os próprios autores.

Revisão Bibliométrica sobre Veículos Autônomos. Na RB o tempo da pesquisa foi limitado até dezembro de 2019, utilizando-se as bases de dados da Web of Science e Scopus, sendo que as buscas foram realizadas no dia 24 de março de 2020 e somente documentos em inglês. A pesquisa na Web of Science foi limitada pelas áreas Transportation Science Technology e Transportation, na Scopus foi pela área Social Sciences.
A string de pesquisa na Web of Science foi: (("autonomous" $O R$ "automated" $O R$ "self-driving" OR "driverless") NEAR/O ("vehicle\$" OR "car\$" OR "driving" OR "automobile\$" $O R$ "bus*" $O R$ "taxi\$" $O R$ "truck\$" OR "electric vehicle\$" OR "road vehicle\$")) $O R$ (("connected") NEAR/1 ("automated" OR "autonomous") AND ("vehicle\$" OR "car\$" OR "driving" OR "transportation")) $O R$ "cooperative intelligent transport 
system\$" OR "cooperative-ITS", sendo encontrados 3.926 resultados com 29.409 citações ao todo.

Já a string de pesquisa na Scopus foi semelhante, somente alterando operadores de pesquisa próprios da base: (("autonomous" $O R$ "automated" $O R$ "selfdriving" OR "driverless") w/O ("vehicle $\$$ " OR "car\$" OR "driving" OR "automobile\$" $O R$ "bus*" $O R$ "taxi\$" $O R$ "truck\$" OR "electric vehicle\$" OR "road vehicle\$")) $O R$ (("connected") w/1 ("automated" OR "autonomous") AND ("vehicle\$" OR "car\$" OR "driving" OR "transportation")) $O R$ "cooperative intelligent transport system $\$$ " OR "cooperative-ITS", sendo encontrados 3.047 resultados com 19.707 citações.

Evolução das publicações e citações até 2019. Na Figura 2 apresenta-se a evolução porcentual do tema, em que em ambas as bases às publicações e citações vêm crescendo consideravelmente ano a ano, com maior intensidade a partir de 2014.

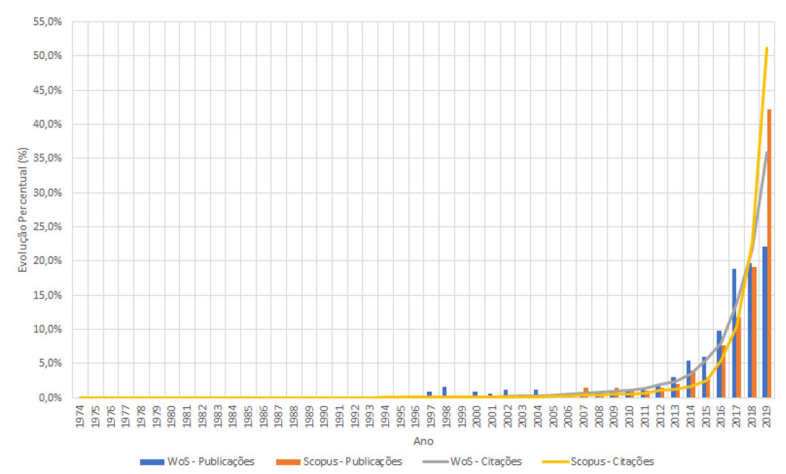

Figura 2. Evolução do número de publicações e citações na Web of Science e Scopus

Fonte: Os próprios autores, baseado nos dados extraídos da Web of Science e Scopus.
Na Web of Science as publicações iniciam-se em 1979 e na Scopus em 1974, e vão até dezembro de 2019. Na evolução das publicações observa-se que na Web of Science cerca de 60\% ocorreram de 2017 a 2019, enquanto que na Scopus somente 2019 é responsável por mais de $40 \%$ de suas publicações. Já em relação a evolução das citações, na Web of Science quase $60 \%$ das suas citações foram em 2018 e 2019, enquanto na Scopus mais de 50\% foram somente em 2019.

\section{Principais periódicos dos documentos} publicados. Ao se analisar os dados da pesquisa na Web of Science, existem 3.926 documentos com 29.409 citações ao todo, publicados em 343 periódicos no período de 1979 a dezembro de 2019. Neste período os 10 periódicos que mais publicaram sobre o tema de VAs representam $43 \%$ de todas as publicações nesta base de dados (1.688 documentos). Já os 10 periódicos que mais possuem citações sobre o tema representam $64,3 \%$ das citações (18.898 citações).

Na Figura 3, observa-se o crescimento do número de documentos nos últimos 20 anos (1999 a 2019) nos 10 periódicos que mais publicaram sobre VAs, representando 99,3\% (1.677 documentos). E na Figura 4 está o crescimento do número de citações nos 10 periódicos que mais possuem citações sobre o tema nos últimos 20 anos (1999 a 2019), representando $99,7 \%$ das citações destes periódicos (18.844 citações).

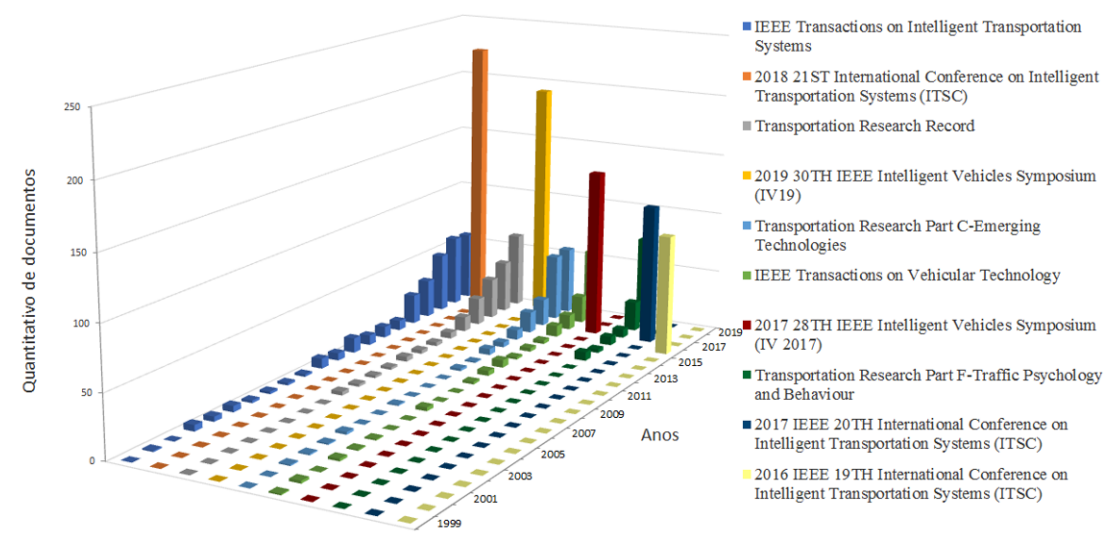

Figura 3. Crescimento do número de documentos nos periódicos que mais publicaram sobre o tema de 1999 a 2019 na Web of Science.

Fonte: Os próprios autores, baseado nos dados extraídos da Web of Science. 

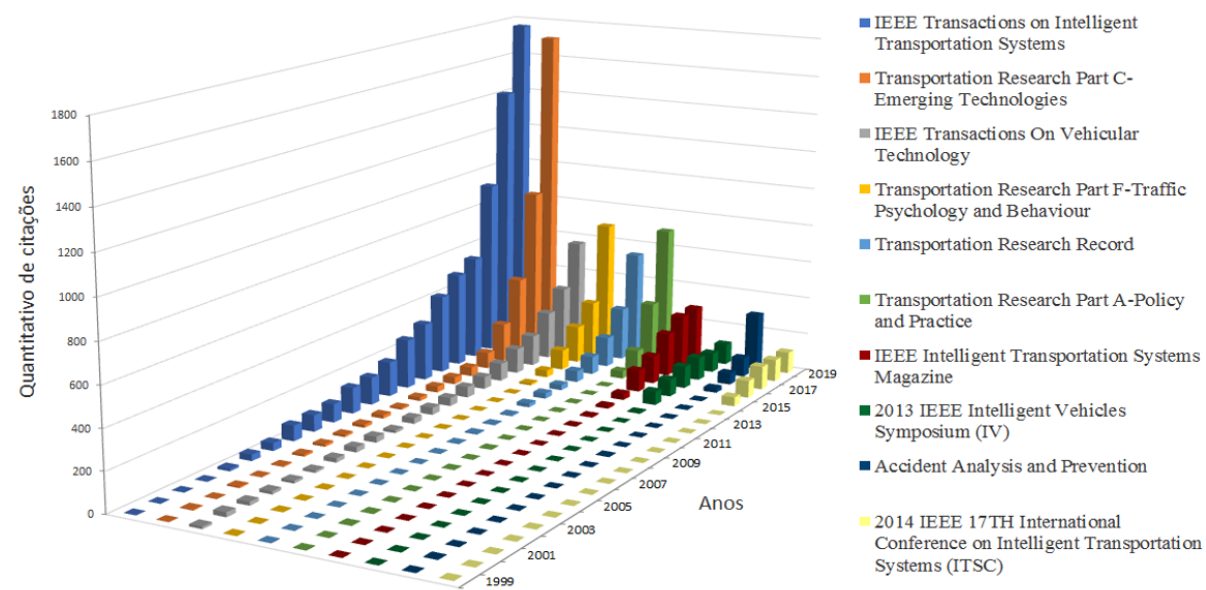

Figura 4. Crescimento do número de citações nos periódicos que mais possuem citações sobre o tema no período de 1999 a 2019 na Web of Science

Fonte: os próprios autores, baseado nos dados extraídos da Web of Science.

A revista IEEE Transactions on Intelligent Transportation Systems, além de ser a que mais publicou sobre o tema, também é aquela que apresentou o maior número de citações. E a revista Transportation Research Record apesar de ser a terceira em número de publicações, é a quinta no número de citações.

Em relação as revistas que mais publicam e/ou citam sobre o tema de VAs, na Web of Science, é também pertinente analisar sua posição no ranque do Journal Citation Reports, os seus fatores de impacto (Journal Impact Factor - JIF) e o quartil do fator de impacto (Journal Impact Factor Quartile). Este ranque possui 73 revistas nas categorias de Transportation Science Technology e Transportation (Clarivate Analytics, 2020). Assim, na Tabela 1, percebe-se que a revista IEEE Transactions on Intelligent Transportation Systems além de ser a que mais publicou e possuiu citações sobre o tema, apresenta o maior fator de impacto entre as revistas apresentadas, ocupando o quartil Q1, sendo a quinta no ranque. E a revista Transportation Research Record apesar de ser uma das que mais publicou e citou sobre o tema, não apresenta um bom fator de impacto, com a $64^{\circ}$ posição no ranque, estando no quartil Q4.

Já ao se analisar os dados da pesquisa na Scopus, existem 3.047 documentos com 19.707 citações ao todo, publicados em 629 periódicos no período de 1974 a dezembro de 2019. Sendo que os 10 periódicos que mais publicaram representam $32,8 \%$ de todas as publicações ( 1.000 documentos) e os 10 periódicos que mais possuem citações representam 61,6\% (12.136 citações).
Tabela 1. Classificação no JCR das revistas que mais publicaram e citaram sobre o tema na Web of Science.

\begin{tabular}{|c|c|c|c|c|c|}
\hline Nome do periódico & 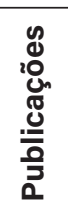 & 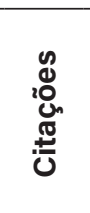 & 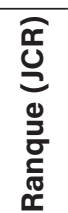 & 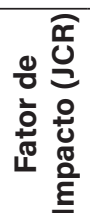 & 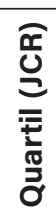 \\
\hline $\begin{array}{c}\text { IEEE Transactions on } \\
\text { Intelligent Transportation }\end{array}$ & 291 & 6929 & $5^{\circ}$ & 6,319 & Q1 \\
\hline $\begin{array}{c}\text { Systems } \\
\text { Transportation Research } \\
\text { Part C - Emerging } \\
\text { Technologies }\end{array}$ & 175 & 3504 & $6^{\circ}$ & 6,077 & Q1 \\
\hline $\begin{array}{l}\text { IEEE Transactions on } \\
\text { Vehicular Technology }\end{array}$ & 139 & 2013 & $8^{\circ}$ & 5,379 & Q1 \\
\hline $\begin{array}{l}\text { Transportation Research } \\
\text { Part A - Policy and Practice }\end{array}$ & 53 & 1189 & $18^{\circ}$ & 3,992 & Q1 \\
\hline $\begin{array}{c}\text { Accident Analysis and } \\
\text { Prevention } \\
\text { IEEE Intelligent }\end{array}$ & 49 & 498 & $21^{\circ}$ & 3,655 & Q1 \\
\hline $\begin{array}{c}\text { Transportation Systems } \\
\text { Magazine } \\
\text { Transportation Research }\end{array}$ & 50 & 1116 & $25^{\circ}$ & 3,363 & Q2 \\
\hline $\begin{array}{c}\text { Part F - Traffic Psychology } \\
\text { and Behaviour }\end{array}$ & 121 & 1325 & $33^{\circ}$ & 2,518 & Q2 \\
\hline $\begin{array}{c}\text { Transportation Research } \\
\text { Record }\end{array}$ & 194 & 1226 & $64^{\circ}$ & 1,029 & Q4 \\
\hline
\end{tabular}

Fonte: Os próprios autores, baseado nos dados extraídos da Web of Science e classificação de Clarivate Analytics (2020).

Na Figura 5, mostra-se o crescimento do número de documentos nos últimos 20 anos (1999 a 2019) publicados nos 10 periódicos que mais publicaram na Scopus sobre VAs, representando $99,8 \%$ de suas publicações (998 documentos). A Figura 6, contém o crescimento do número de citações nos 10 periódicos que mais possuem citações sobre o tema nos últimos 20 anos (1999 a 2019), referente aos dados da Scopus, representando $100 \%$ das citações destes periódicos (12.136 citações). 


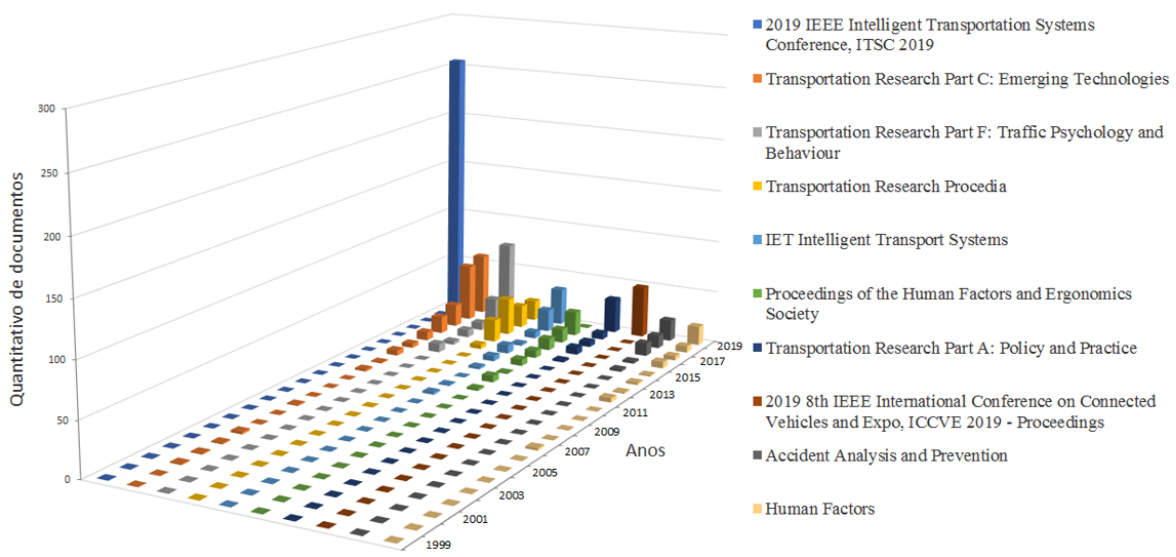

Figura 5. Crescimento do número de documentos nos periódicos que mais publicam sobre o tema no período de 1999 a 2019 na Scopus.

Fonte: Os próprios autores, baseado nos dados extraídos da Scopus.

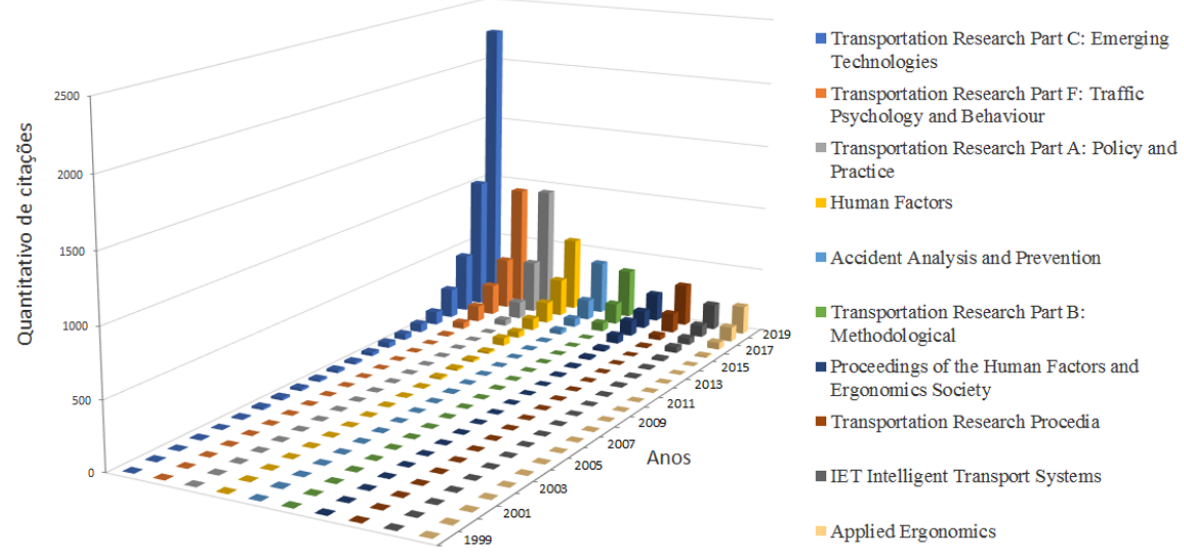

Figura 6. Crescimento do número de citações nos periódicos que mais possuem citações sobre o tema de 1999 a 2019 na Scopus.

Fonte: Os próprios autores, baseado nos dados extraídos da Scopus.

A revista Transportation Research Part C: Emerging Technologies é a segunda que mais publicou sobre o tema e está em primeiro no número de citações. $\mathrm{E}$ a Transportation Research Part F: Traffic Psychology and Behaviour é a terceira em número de publicações e segunda em citações, configurandose nas duas revistas que mais publicaram e citaram sobre este tema.

Além de identificar o número de publicações e citações nas revistas, também foi avaliada a sua posição no ranque da SCImago Journal (SJR), o fator de impacto e o quartil a que pertence. Na subárea
Transportation da área Social Sciences existem 149 periódicos entre journals, book series, conferences and proceedings e trade journals (Scimago Institutions Rankings, 2019). Como apresentado na Tabela 2, a revista Transportation Research Part C: Emerging Technologies possui o melhor fator de impacto, ficando em segundo lugar no ranque, e situada no quartil Q1. Já a IET Intelligent Transport Systems, terceira em número de publicações e nona em citações, o seu fator de impacto fica na $46^{\circ}$ posição, estando no quartil Q2. 
Tabela 2. Classificação no SJR das revistas que mais publicaram e citaram sobre o tema na Scopus.

\begin{tabular}{|c|c|c|c|c|c|}
\hline Nome do periódico & 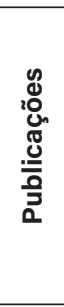 & 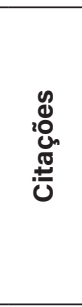 & 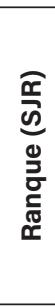 & 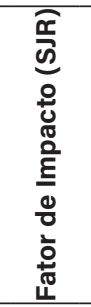 & 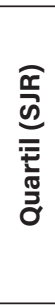 \\
\hline $\begin{array}{c}\text { Transportation Research Part C: } \\
\text { Emerging Technologies }\end{array}$ & 176 & 4363 & $2^{\circ}$ & 3,342 & Q1 \\
\hline $\begin{array}{c}\text { Transportation Research Part B: } \\
\text { Methodological }\end{array}$ & 44 & 598 & $6^{\circ}$ & 2,895 & Q1 \\
\hline $\begin{array}{c}\text { Transportation Research Part A: } \\
\text { Policy and Practice }\end{array}$ & 53 & 1528 & $10^{\circ}$ & 2,109 & Q1 \\
\hline $\begin{array}{l}\text { Transportation Research Part F: } \\
\text { Traffic Psychology and Behaviour }\end{array}$ & 122 & 1749 & $28^{\circ}$ & 1,146 & Q1 \\
\hline IET Intelligent Transport Systems & 82 & 429 & $46^{\circ}$ & 0,627 & Q2 \\
\hline Transportation Research Procedia & 100 & 519 & $56^{\circ}$ & 0,476 & - \\
\hline
\end{tabular}

Acoplamento bibliográfico. $\mathrm{O}$ acoplamento bibliográfico ocorre quando dois documentos citam um mesmo documento, isto é, os documentos possuem citações em comum. Para determinar o acoplamento bibliográfico, é realizada uma sobreposição das listas de referências das publicações, buscando referencias em comum, e assim quanto maior o número de referências que duas publicações têm em comum, mais forte é a relação de acoplamento bibliográfico entre elas (Kessler, 1963; van Eck e Waltman, 2014).

Para a criação do mapa de acoplamento bibliográfico no VOSviewer, foi selecionado o método de contagem completa, em que cada link de acoplamento bibliográfico tem o mesmo peso. Foram utilizados inicialmente todos os documentos das pesquisas das bases de dados. Em seguida se calcula de todos os documentos a força total dos links com os outros documentos, selecionando-se somente os 1000 documentos com maior força total do link. Na Figura 7 é apresentado o acoplamento bibliográfico dos dados da pesquisa na Web of Science (WoS), agrupados em quatro cluster. O cluster 1 WoS (vermelho) é voltado para a área de aceitabilidade referente aos VAs. No cluster 2 WoS (verde) há alguns estudos dos possíveis impactos da direção automatizada no sistema de transporte. Já o cluster 3 WoS (azul) reúne estudos da operação e desenvolvimento de VAs. E no cluster 4 WoS (amarelo) agrega trabalhos sobre simuladores de direção.

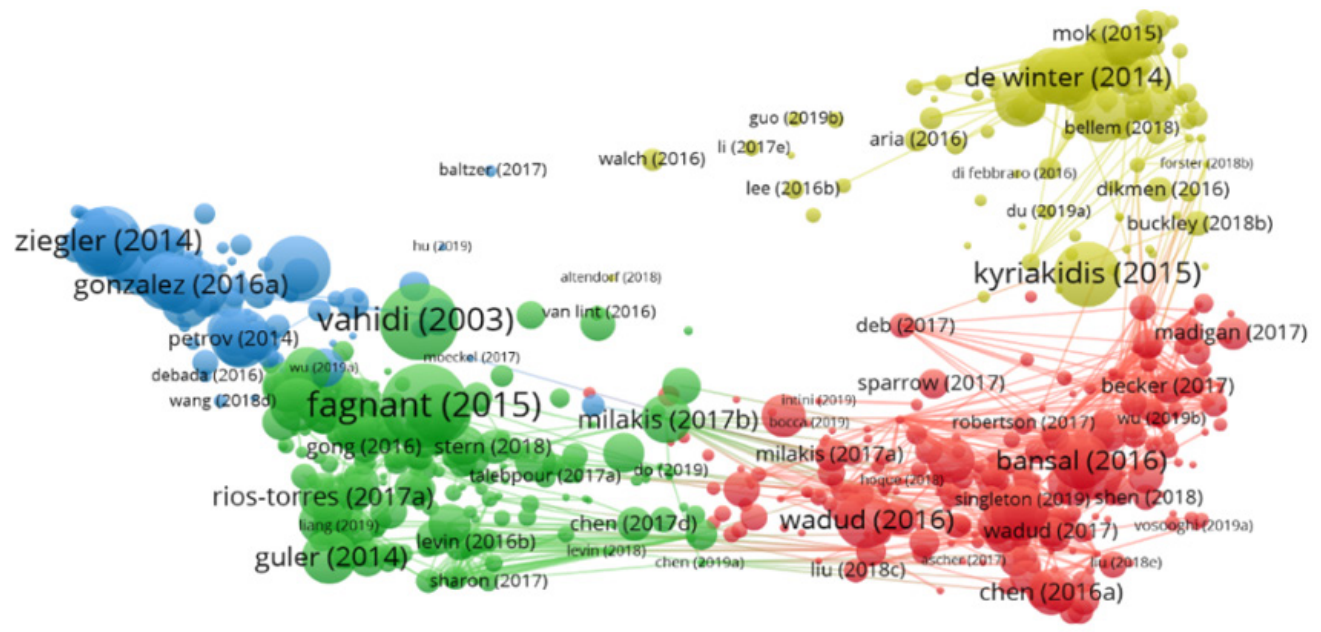

Figura 7. Acoplamento bibliográfico com os 1000 documentos com maior força total de link com os demais documentos - Web of Science.

Fonte: Os próprios autores, baseado nos dados extraídos da Web of Science. 
O acoplamento bibliográfico dos dados da pesquisa na Scopus, com seis clusters, apresentase na Figura 8. O cluster 1 Sco (vermelho) possui semelhança com o cluster 4 WoS, voltado para simuladores de direção. Já o cluster 2 Sco (verde) assemelha-se ao cluster 2 WoS, com estudos sobre tráfego misto, veículos movidos por humanos e autônomos. Os clusters 3 Sco (azul escuro) e 4 Sco (amarelo) possuem semelhanças com o cluster 1 WoS, focados na adoção e aceitabilidade de VAs. Já o cluster 5 Sco (roxo) aborda sobre questões éticas e sociais, e o cluster 6 Sco (azul claro) com 8 documentos, focados no enjoo de movimento causado pelo uso dos VA.

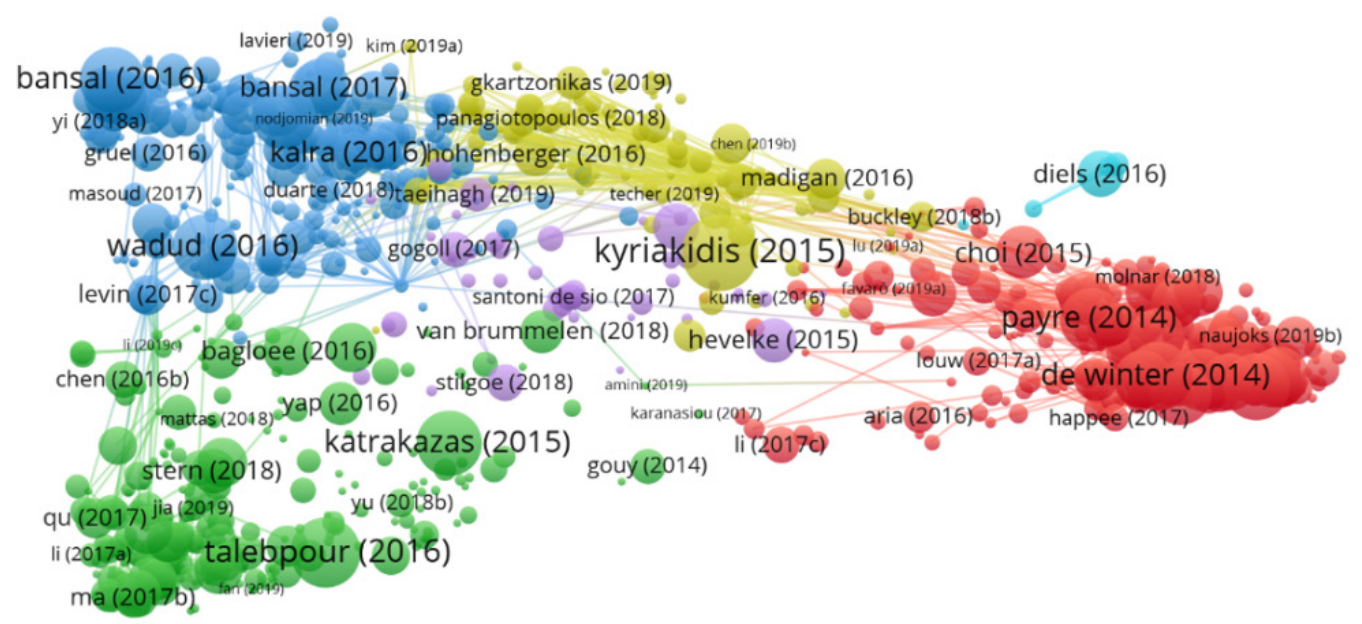

Figura 8. Acoplamento bibliográfico com os 1000 documentos com maior força total de link com os demais documentos - Scopus.

Fonte: Os próprios autores, baseado nos dados extraídos da Scopus.

Publicações por países. Conforme mostrado na Figura 9, nos documentos da Web of Science, o primeiro lugar ficou com os Estados Unidos (29,1\%), seguido da Alemanha (20,1\%), China (13,5\%), França $(6,1 \%)$ e Reino Unido (5,1\%). No caso da Scopus tem-se novamente os Estados Unidos $(30,4 \%)$ em primeiro lugar, seguido novamente da Alemanha $(15,6 \%)$ e China $(10,5 \%)$, e logo o Reino Unido (7,5\%) e Países Baixos (5,8\%). Com relação aos países em desenvolvimento, destacam-se em número de publicações, a China, Brasil (0,91\% na Web of Science e 0,65\% na Scopus), Rússia (0,66 e 0,68\%) e Índia (0,86 e 1,05\%).

Considerando os clusters do acoplamento bibliográfico, na Figura 10 são apresentados os resultados da Web of Science, com as linhas de pesquisas que estão sendo desenvolvidas em cada país. Os Estados Unidos lideram nos cluster 1 WoS e 2 Wos (42,47 e 55,90\%, respectivamente), e está em terceiro lugar no 3 WoS $(15,06 \%)$ e segundo no 4 WoS (18,18\%). Já a Alemanha lidera os clusters 3 WoS e 4 WoS $(23,94$ e $37,66 \%)$, e está em terceiro no cluster 1 WoS $(10,37 \%)$ e em sexto lugar no cluster 2 WoS (4,86\%). Em relação aos países em desenvolvimento, a China aparece nos quatro clusters, principalmente nos clusters 1 WoS, 2 WoS e 3 WoS, ocupando o segundo lugar (11,37; 27,78 e $21,62 \%$ respectivamente). A Rússia apesar de apresentar 26 publicações na análise da Web of Science, seus documentos não foram categorizados nos clusters. A Índia aparece nos clusters 1 WoS, 2 WoS e 3 WoS (0,67; 1,04 e 1,16\%), o Brasil nos clusters 1 WoS e 2 WoS (0,67 e 0,35\%). O cluster 4 WoS, voltado para simuladores de direção, contempla unicamente a China com 2,60\% das publicações.

Para o caso dos clusters de acoplamento bibliográfico da Scopus nos países, na Figura 11, observa-se a distribuição percentual dos documentos publicados. O cluster 6 Sco, possui apenas seis países, sendo que somente os Países Baixos e Reino Unido (representando 62,5\% cada país), têm a maior percentagem, e os demais países (Austrália, Alemanha, Malásia e Estados Unidos) possuem somente uma publicação neste cluster (12,5\%), considerando as possíveis co-autoria. 
Romano \& Taco - Veículo Autônomo: Uma Visão Geral da Produção Científica baseada na Análise Bibliométrica

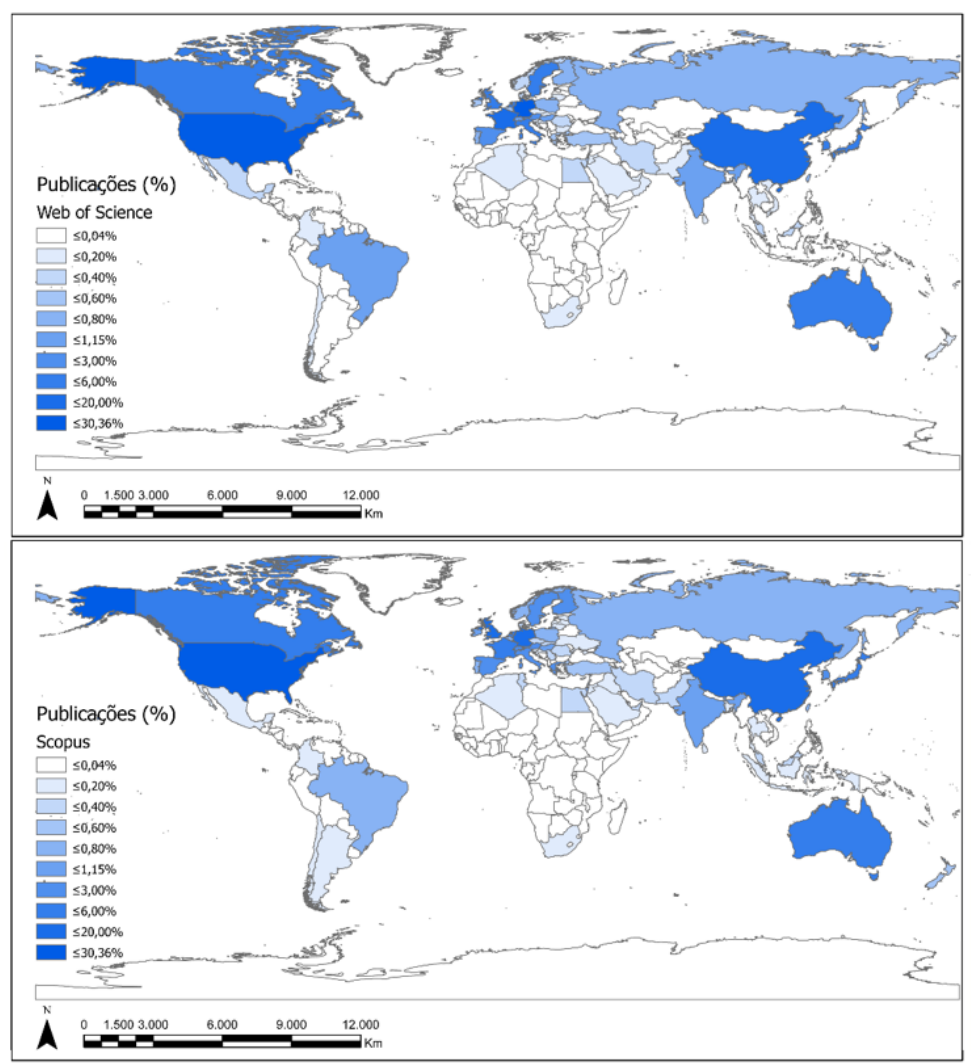

Figura 9. Países que mais publicaram na Web of Science (acima) e Scopus (abaixo). Fonte: Os próprios autores, baseado nos dados extraídos da Web of Science e Scopus.

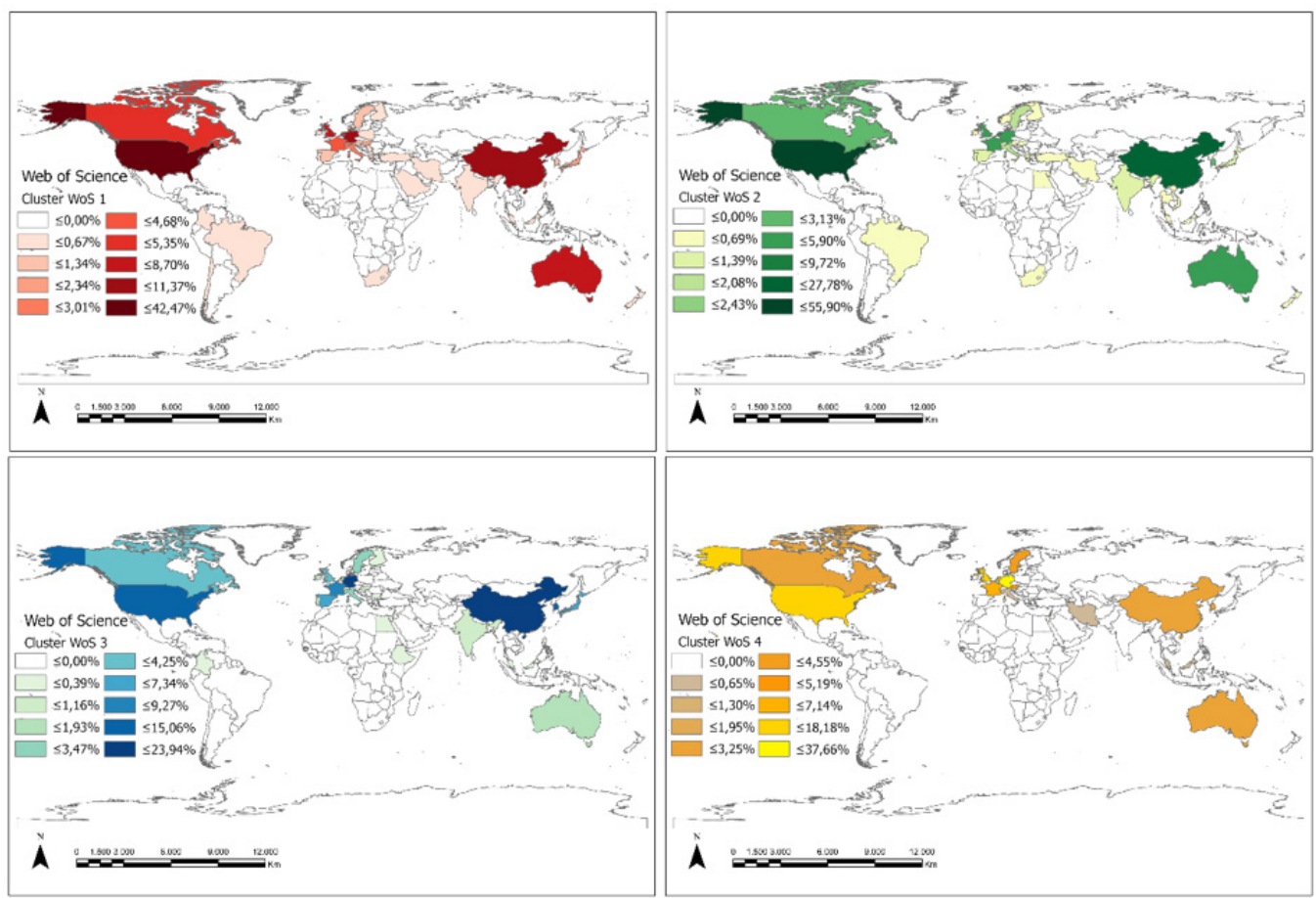

Figura 10. Países que mais publicaram em cada cluster do acoplamento bibliográfico da Web of Science. Fonte: Os próprios autores, baseado nos dados extraídos da Web of Science. 




Figura 11. Países que mais publicaram em cada cluster do acoplamento bibliográfico da Scopus. Fonte: Os próprios autores, baseado nos dados extraídos da Scopus.

Com exceção do cluster 6 Sco, os Estados Unidos lideram todos os clusters em número de documentos publicados, $1 \mathrm{Sco}, 2 \mathrm{Sco}, 3 \mathrm{Sco}, 4 \mathrm{Sco}$ e $5 \mathrm{Sco}(31,86$; 44,$53 ; 45,35 ; 26,04$ e $24,14 \%$, respectivamente). E a Alemanha se encontra em segundo lugar nos clusters 1 Sco, 4 Sco e 5 Sco (25,76; 17,71 e 20,69\%), e em terceiro nos 2 Sco e 3 Sco (10,95 e 10,41\%). Em relação aos países em desenvolvimento, a China tem uma boa representatividade nos clusters $1 \mathrm{Sco}$, 2 Sco, 3 Sco e 4 Sco $(3,73,27,74,7,06$ e 9,38\%), e com uma publicação no cluster 5 Sco (1,72\%). O cluster 3 Sco congrega três documentos da Índia, Brasil e Rússia, representado 1,12\% cada país. Já o cluster 2 Sco possui publicações da Índia (0,36\%), e os clusters 1 Sco $(0,67 \%)$ e 5 Sco $(1,72 \%)$ contém documentos brasileiros.

\section{Considerações Finais}

Este estudo teve como objetivo levantar o estado da arte referente a VA, observando o desenvolvimento do tema e analisando os periódicos que mais publicaram e citaram sobre o tema, as linhas de pesquisa e a distribuição de publicação nos países. 
Com este levantamento, foi notado que o tema de VAs teve um crescimento considerável ano a ano nas duas bases de dados, principalmente na Scopus, com maior intensidade a partir de 2014, refletindo o aumento do interesse pelo assunto no decorrer dos anos.

Nos documentos da Web of Science a revista IEEE Transactions on Intelligent Transportation Systems foi a que mais publicou e citou sobre os VAs. Além disso, este periódico apresenta o maior fator de impacto entre todos nos dados da Web of Science, estando em quinto lugar no JCR. Já nos dados da Scopus, o periódico que apresentou maior relevância foi a revista Transportation Research Part C: Emerging Technologies, que é o segundo que mais publicou e o primeiro em número de citações, apresentando o maior fator de impacto entre os analisados, estando em segundo lugar no SJR.

Nas análises de acoplamento bibliográfico foram identificadas duas grandes linhas de pesquisa de VAs, uma voltada aos aspectos tecnológicos, focando na direção automatizada no sistema de transporte e operação dos VAs (2 WoS, 3 WoS e 2 Sco), e a outra para a questão social, relacionada aos aspectos comportamentais e de aceitabilidade dos VA (1 WoS, 4 WoS, 1 Sco, 3 Sco, 4 Sco e 5 Sco e 6 Sco).

$\mathrm{Na}$ distribuição de publicações por países é perceptível que os Estados Unidos, Alemanha e China lideram o número de publicações nas duas bases de dados. Ao se considerar a representatividade dos países em desenvolvimento aparecem a China, Brasil, Rússia e Índia. Desses, a China que se encontra em segundo lugar em número de publicações, compete com os países desenvolvidos. Quanto as linhas de pesquisa voltada para os aspectos tecnológicos, os países que lideram são principalmente os Estados Unidos e a China. Na linha referente às questões sociais se destacam os Estados Unidos e Alemanha.

Visto os resultados obtidos, foi gerado um melhor entendimento sobre as pesquisas envolvendo os VAs, que podem ser resumidas em duas áreas de conhecimento dos transportes, da oferta e da demanda. Do lado da oferta, as linhas de pesquisa focam-se nos aspectos técnicos e tecnológicos, enquanto do lado da demanda estão sendo estudadas questões sociais e comportamentais do usuário perante a implantação dos VAs.

Dessa forma, constata-se a vantagem dos países desenvolvidos em implantar os VAs, pois são detentores da tecnologia necessária para a produção de veículos totalmente autônomos, que não precisem de nenhuma condução humana. No caso dos países em desenvolvimento, como - Brasil, um processo de interação com os países líderes identificados poderá auxiliar na implantação dos VAs no contexto nacional.

Em relação as questões sociais e comportamentais, seja nos países desenvolvidos ou em desenvolvimento, as barreiras de implantação sempre estarão presentes, principalmente em relação a segurança e privacidade. Isto mostra que são necessários estudos focando na aceitabilidade do usuário e comportamento do motorista.

\section{Agradecimentos}

Um agradecimento à Coordenação de Aperfeiçoamento de Pessoal de Nível Superior - Brasil (CAPES), pela concessão da bolsa de estudos ao primeiro autor para o desenvolvimento da dissertação que deu origem a este trabalho. Também agradecemos ao nosso Grupo de Pesquisa Comportamento em Transportes e Novas Tecnologias (CTNT) do Programa de PósGraduação em Transportes (PPGT) da Universidade de Brasília (UnB).

\section{REFERENCIAS}

Anderson, J. M., Kalra, N., Stanley, K. D., Sorensen, P., Samaras, C., \& Oluwatola, O. A. (2014). Autonomous vehicle technology a guide for policymakers. In RAND Corporation.

Clarivate Analytics. (2020). InCites Journal Citation Reports. dataset updated Jun 29, 2020. Recuperado em 5 de setembro, 2020, de https://jcr.clarivate.com/JCRJournalHomeAction.action 
Fagnant, D. J., \& Kockelman, K. (2015). Preparing a nation for autonomous vehicles: Opportunities, barriers and policy recommendations. Transportation Research Part A: Policy and Practice, 77, 167-181. https:// doi.org/10.1016/j.tra.2015.04.003

Gandia, R. M., Antonialli, F., Cavazza, B. H., Neto, A. M., Lima, D. A. de, Sugano, J. Y., Nicolai, I., \& Zambalde, A. L. (2019). Autonomous vehicles: scientometric and bibliometric review. Transport Reviews, 39(1), 9-28. https://doi.org/10.1080/01441647.2018.1518937

Gogoll, J., e Müller, J. F. (2017) Autonomous Cars: In Favor of a Mandatory Ethics Setting. Science and Engineering Ethics, 23(3), 681-700. doi:10.1007/s11948-016-9806-x

Hevelke, A., e Nida-Rümelin, J. (2015) Responsibility for Crashes of Autonomous Vehicles: An Ethical Analysis. Science and Engineering Ethics, 21(3), 619-630. doi:10.1007/s11948-014-9565-5

Kessler, M. M. (1963). Bibliographic coupling between scientific papers. American Documentation, 14(1), 1025. https://doi.org/10.1002/asi.5090140103

Li, Y., Wang, Y., Rui, X., Li, Y., Li, Y., Wang, H., Zuo, J., \& Tong, Y. (2017). Sources of atmospheric pollution: a bibliometric analysis. Scientometrics, 112(2), 1025-1045. https://doi.org/10.1007/s11192-017-2421-Z

Milakis, D., Van Arem, B., \& Van Wee, B. (2017). Policy and society related implications of automated driving: A review of literature and directions for future research. Journal of Intelligent Transportation Systems: Technology, Planning, and Operations, 21(4), 324-348. https://doi.org/10.1080/15472450.2017.1291351

Modak, N. M., Merigó, J. M., Weber, R., Manzor, F., \& Ortúzar, J. de D. (2019). Fifty years of Transportation Research journals: A bibliometric overview. Transportation Research Part A: Policy and Practice, 120, 188-223. https://doi.org/10.1016/j.tra.2018.11.015

Petit, J., e Shladover, S. E. (2015) Potential Cyberattacks on Automated Vehicles. IEEE Transactions on Intelligent Transportation Systems, 16(2), 546-556. doi:10.1109/TITS.2014.2342271

Romano, A. B., Taco, P. W. G., Mariano, A. M., \& Feitosa, Z. O. (2018). Revisão Bibliométrica Dos Fatores Que Influenciam O Uso De Bicicleta Fazendo Uso Da Teoria Do Enfoque Meta Analítico Consolidado (TEMAC). $32^{\circ}$ Congresso de Pesquisa e Ensino em Transporte da ANPET, 2726-2737.

Santoni de Sio, F. (2017) Killing by Autonomous Vehicles and the Legal Doctrine of Necessity. Ethical Theory and Moral Practice, 20(2), 411-429. doi:10.1007/s10677-017-9780-7

Scimago Institutions Rankings. (2019). Scimago Journal \& Country Rank. Recuperado em 5 de setembro, 2020, de https://www.scimagojr.com/journalrank.php?category=3313\&area=3300\&year=2019

The International Association of Public Transport. (2017). Policy brief - Autonomous vehicles: a potential game changer for urban mobility. The International Association of Public Transport - UITP. Recuperado de https://www.uitp.org/publications/autonomous-vehicles-a-potential-game-changer-for-urbanmobility/

van Eck, N. J., \& Waltman, L. (2014). Visualizing Bibliometric Networks. In Measuring Scholarly Impact (p. 285-320). Springer International Publishing. https://doi.org/10.1007/978-3-319-10377-8_13

Waltman, L., van Eck, N. J., \& Noyons, E. C. M. (2010). A unified approach to mapping and clustering of bibliometric networks. Journal of Informetrics, 4(4), 629-635. https://doi.org/0.1016/j.joi.2010.07.002 